\title{
Preliminary Design Study of the TMT Telescope Structure System: Overview
}

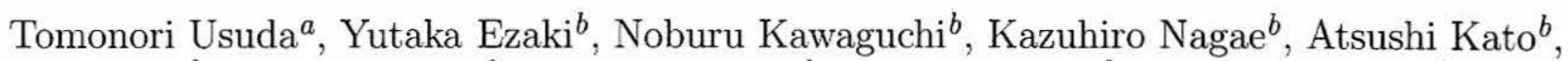
Junji Takaki ${ }^{b}$, Masaki Hirano ${ }^{b}$, Tomoya Hattori ${ }^{b}$, Masaki Tabata ${ }^{b}$, Yasushi Horiuchi ${ }^{b}$, Yusuke Saruta $^{b}$, Satoru Sofuku ${ }^{b}$, Noboru Itoh $^{b}$, Takeharu Oshima ${ }^{b}$, Takashi Takanezawa ${ }^{b}$, Makoto Endo $^{b}$, Junji Inatani ${ }^{a}$, Masanori Iye ${ }^{a}$, Amir Sadjadpour $^{c}$, Mark Sirota $^{c}$, Scott Roberts $^{c}$ and Larry Stepp ${ }^{c}$

${ }^{a}$ National Astronomical Observatory of Japan, 2-21-1 Osawa, Mitaka, Tokyo, Japan;

${ }^{b}$ Mitsubishi Electric Corporation, 8-1-1, Tsukaguchi-Honmachi, Amagasaki, Hyogo, Japan;

${ }^{c}$ Thirty Meter Telescope Project, 1111 South Arroyo Parkway, Pasadena, CA, U.S.A.

\begin{abstract}
We present an overview of the preliminary design of the Telescope Structure System (STR) of Thirty Meter Telescope (TMT). NAOJ was given responsibility for the TMT STR in early 2012 and engaged Mitsubishi Electric Corporation (MELCO) to take over the preliminary design work. MELCO performed a comprehensive preliminary design study in 2012 and 2013 and the design successfully passed its Preliminary Design Review (PDR) in November 2013 and April 2014. Design optimizations were pursued to better meet the design requirements and improvements were made in the designs of many of the telescope subsystems as follows:
\end{abstract}

1. 6-legged Top End configuration to support secondary mirror (M2) in order to reduce deformation of the Top End and to keep the same $4 \%$ blockage of the full aperture as the previous STR design.

2. "Double Lower Tube" of the elevation (EL) structure to reduce the required stroke of the primary mirror (M1) actuators to compensate the primary mirror cell (M1 Cell) deformation caused during the EL angle change in accordance with the requirements.

3. M1 Segment Handling System (SHS) to be able to make removing and installing 10 Mirror Segment Assemblies per day safely and with ease over M1 area where access of personnel is extremely difficult. This requires semi-automatic sequence operation and a robotic Segment Lifting Fixture (SLF) designed based on the Compliance Control System, developed for controlling industrial robots, with a mechanism to enable precise control within the six degrees of freedom of position control.

4. $\mathrm{CO}_{2}$ snow cleaning system to clean M1 every few weeks that is similar to the mechanical system that has been used at Subaru Telescope.

5. Seismic isolation and restraint systems with respect to safety; the maximum acceleration allowed for M1, M2, tertiary mirror (M3), LGSF, and science instruments in 1,000 year return period earthquakes are defined in the requirements.

The Seismic requirements apply to any EL angle, regardless of the operational status of Hydro Static Bearing (HSB) system and stow lock pins. In order to find a practical solution, design optimization study for seismic risk mitigation was carried out extensively, including the performing of dynamic response analyses of the STR system under the time dependent acceleration profile of seven major earthquakes. The work is now moving to the final design phase from April 2014 for two years.

Keywords: Astronomy, Ground based Extremely Large Telescope (ELT), Telescope Structure, Mount Control System, Segment Handling System, Seismic risk mitigation

Further author information: (Send correspondence to T.U.)

T.U.: E-mail: usuda@naoj.org, Telephone: 8142234 3522, Address: 2-21-1 Osawa, Mitaka, Tokyo 181-8588, Japan

Ground-based and Airborne Telescopes V, edited by Larry M. Stepp, Roberto Gilmozzi,

Helen J. Hall, Proc. of SPIE Vol. 9145, 91452F - (c) 2014 SPIE

CCC code: $0277-786 \mathrm{X} / 14 / \$ 18 \cdot$ doi: $10.1117 / 12.2055767$ 


\section{INTRODUCTION}

Ground based Extremely Large Telescope (ELT) has emerged from an international heritage of 8-10 m class telescopes like Keck I and II, Gemini, VLTs, and Subaru Telescope. Thirty Meter Telescope project (TMT) is an international partnership whose mission is to build a $30 \mathrm{~m}$, finely-segmented filled-aperture optical-infrared telescope on the summit of Mauna Kea, on the island of Hawai'i. TMT will have a multi-conjugate adaptive optics (MCAO) system that delivers diffraction-limited images in the infrared down to a wavelength of approximately $1 \mu \mathrm{m}$. Multiple science instruments on the Nasmyth platforms will be addressed by a steerable tertiary mirror, allowing efficient instrument switching during the night and rapid follow-up of transient events. The telescope structure is an altitude-azimuth design with a single azimuth track and two large elevation journals. The telescope uses direct drive motors, hydrostatic bearings and tape encoders. The observing range is from 1 to 65 degrees zenith angle. The telescope can rotate continuously from -250 to +250 degrees azimuth angle without unwrapping the cables. The elevation structure can tilt from the zenith to the horizon for servicing operations. The telescope has a Ritchey-Chretien aplanatic Cassegrain optical design. The TMT $30 \mathrm{~m}$ primary mirror is composed of 492 hexagonal segments. The secondary mirror is a $3.1 \mathrm{~m}$ diameter convex hyperboloid, and the tertiary is a $2.5 \mathrm{~m} \times 3.5 \mathrm{~m}$ flat. The secondary mirror and its structural support present minimal obscuration of the primary, which results in a clean, high contrast and high Strehl ratio point spread function in the adaptive optics (AO) corrected images.

\section{BACKGROUND OF TELESCOPE STRUCTURE DESIGN STUDY}

\subsection{Early Design Stage in 2000s}

Historically, California group developed a $30 \mathrm{~m}$ CELT project, NOAO developed a $30 \mathrm{~m}$ GSMT project, and Canada developed a $20 \mathrm{~m}$ VLOT project, respectively by 2002 . Considering the scale of the projects and based on the Decadal Report 2000 where the importance of building Giant Segmented Mirror Telescopes was highly recognized, the above three groups agreed to form a TMT consortium and established TMT Observatory Corporation in 2003. Productive discussion among the three groups yielded the CELT, GSMT, and VLOT concepts merged into a single $30 \mathrm{~m}$ telescope concept of TMT in 2004. Initial design study of the telescope structure was done by Dynamic Structure Limited (DSL) during 2004-2008. The TMT project office (PO) then took over the DSL design work to refine the initial design. TMT PO produced the Baseline Design by 2011 (Figure 1), where most of the basic design feature was in a concrete form. The Baseline Design was provided to four engineering companies in 2012 to develop cost estimates.

National Astronomical Observatory of Japan (NAOJ) and Mitsubishi Electric Corporation (MELCO) developed independently Japanese Extremely Large Telescope (JELT) project, a three aspheric mirror telescope system with a $30 \mathrm{~m}$ concentric segmented ceramic primary mirror, during 2005-2008. NAOJ, however, decided to join TMT project in 2008 provided that Hawaii was chosen as the construction site. In 2011, the TMT consortium agreed to assign NAOJ the work share for designing and building the Telescope Structure System (STR). Shown in Figure 2 is the current MELCO design described in this paper.

\subsection{NAOJ/MELCO Conceptual Design Study in 2011}

NAOJ and MELCO started conceptual design study in 2011 based on the CAD data and DRD (Design Requirements Document) provided by TMT PO. Start point was design verification studies to confirm if the TMT PO baseline design is compatible with essential parts of DRD. Some areas of concern were identified and possible approaches for revising the design were discussed.

Studies including alternative design solutions based on different concepts were carried out to evaluate the baseline design concept of the telescope structure. Comparative design studies were performed for M2 Spider, Top Ring shape, addition of the Center Section, as well as other areas. Design options were evaluated by comparing the expected stiffness and mass for each design solution. Direct effects of design change were also evaluated for wind loading and thermal performances. In other areas, design improvements were made. For example, the design of the bridge structure of the segment handling system (SHS) and $\mathrm{CO}_{2}$ cleaning system were modified for more ease and safety during operation. 


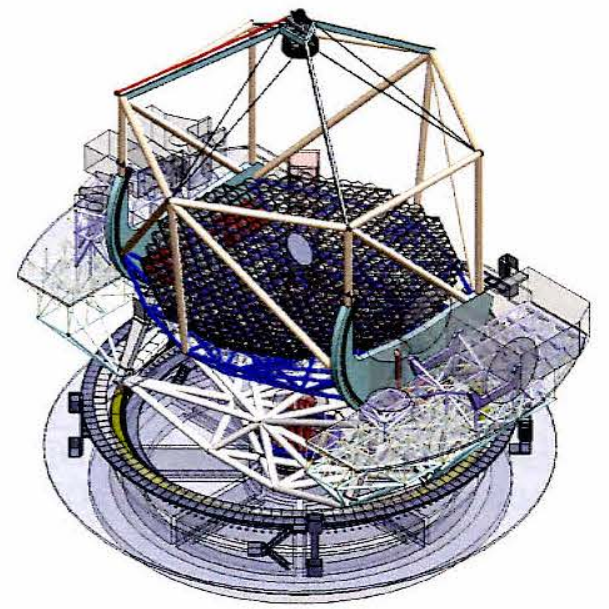

Figure 1. TMT PO Baseline Design in January 2011

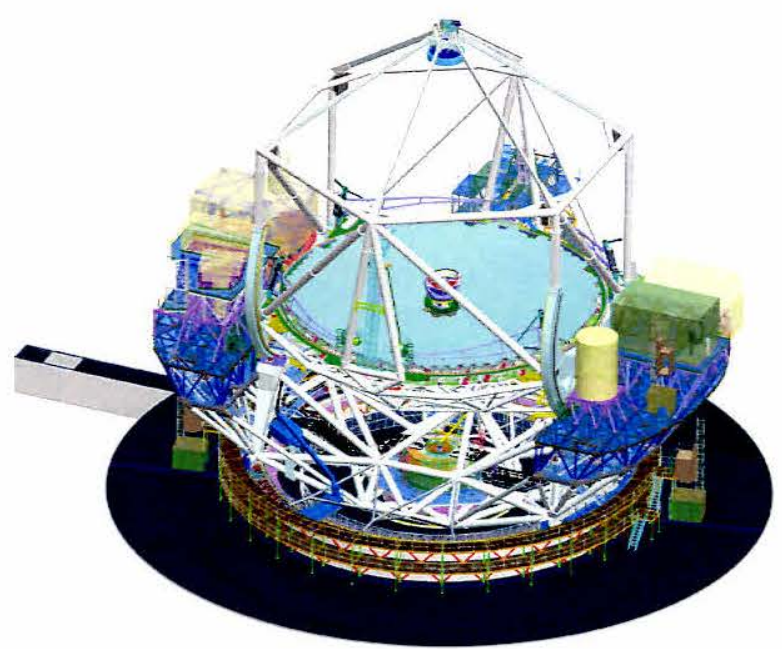

Figure 2. MELCO New Design in September 2013

In addition to these design improvement studies of STR, much effort was paid to define concrete processes for shop assembly tests before shipping and on-site installation. In the scope of the original MELCO plan for shop assembly tests before shipping included testing the basic drive performances of the telescope system by assembling the AZ rail, main structure of the telescope, HSB and the telescope drive system, similarly to the approach MELCO executed for shop assembly test of the Subaru Telescope. However, this requires construction of a large shelter structure to protect the telescope and a sturdy base for measuring the drive performances. NAOJ and TMT PO made decision to abandon this approach in order to avoid cost overrun. Only some of the assembly tests are included in the current plan. For this reason, drive performance test before shipping is not included in the current plan.

Risk analyses for STR construction were made taking advantage of experience gained from Subaru Telescope and ALMA constructions. They include the analyses of effect for any delay in defining the interface control documents (ICDs) and actual delivery of sub-systems by other partners to be integrated with the main telescope structure, schedule delay due to uncontrollable situations on-site, and associated increase in cost.

\subsection{Assumptions Adopted in Preliminary Design Studies}

MELCO design studies are based on DRD and a series of discussion made with NAOJ and TMT PO. The following are some of the basic agreed assumptions which are adopted.

- The stiffness of the ground is assumed at 1,000 MPa. The unpredictable deformation of the AZ rails due to weight load of the telescope structure is assumed to be about $10 \%$.

- The damping factor for dynamic responses of the telescope structure for seismic activities is assumed to be $2 \%$. MELCO originally assumed $1 \%$ for the damping factor but a larger value of $2 \%$ is adopted with the approval of TMT PO and NAOJ. In practice, this made is a significant relaxation of design specification against seismic risks. Note that the damping factor of $2 \%$ is the assumption for the purpose of design study and is not the engineering requirement for implementation.

- The effects from thermal environment and wind loading variation are not included in the scopes of STR design studies because these are the responsibility of the TMT PO. However, design modifications are made to minimize the cross section of the telescope structure to take into account the wind loading. 
- The design study has taken every effort to reduce the mass of the telescope structure without introducing any deterioration in the performance. For any design changes by MELCO involving increase in mass and/or cost is made in consultation with TMT PO and NAOJ.

- In designing the control system, Industrial Standard products are adopted wherever possible.

\subsection{NAOJ and MELCO Preliminary Design Study in 2012-2013}

NAOJ and MELCO moved on to the preliminary design study from 2012 based upon the conceptual study made in 2011. Further design optimization has been pursued to better meet the design requirements and evaluations have been made for key components to be adopted in subsystems.

(1) A "Double Lower Tube" design is introduced in order to reduce the required stroke of M1 actuators to compensate the M1 Cell deformation caused during the EL angle change in accordance with DRD. The Double Lower Tube design is introduced as a more solid design in comparison with the "Virtual spring" design adopted in the TMT PO design to suppress the deformation of M1 Cell.

(2) The number of spiders to support M2 system is increased from 3 to 6 and the tension wires used in the original design were removed. The new design helps to reduce the risk of buckling of spiders under severe seismic events.

(3) With respect to safety, the maximum acceleration allowed for M1, M2, M3, LGSF, and Science Instruments in 1,000 year return period earthquakes are defined in DRD. In addition, STR should not lift up in such a seismic event. These requirements apply to any EL angle, regardless of the operational status of HSB and Stow Lock Pins. To find a practical solution, design optimization study for seismic risk mitigation has been carried out extensively, including the performing of dynamic response analyses of the STR system under the time dependent acceleration profile of 7 major earthquakes.

(4) In order to reduce the scope of shop assembly test before shipping the STR as was agreed in the conceptual design phase study, some countermeasure to verify the subsystem performance to secure total system performance is necessary. Verification procedures during shop test and on-site test have been studied. To shorten the schedule of the on-site test, preparing drive system simulators enabling shop test of control system were proposed. Onsite assembly of STR is necessary to establish an optimum schedule taking into account the limited space and availability of facilities.

\section{KEY DESIGN IMPROVEMENTS}

In this section, several key design improvements are briefly explained.

\subsection{Double Lower Tube}

The baseline design ensured high horizontal stiffness. However, the design also employs a combination of Very Soft Spring and virtual Soft Spring with low vertical stiffness implemented at the joints of Lower Tube and the M1 Cell to reduce the deformation of the M1 Cell to satisfy the stroke requirement for M1 actuators. From engineering point of view, the implementation of virtual soft springs in practice involves risks and an alternative approach to modify the support concept of the M1 Cell was studied. As a result of the study, a design in which the deformation of the M1 Cell is reduced by supporting the M1 Cell with additional supporting members attached at the Lower Tube was adopted. The design is coined "Double Lower Tube" (Fig 3 and 4).

\subsection{6-legged Spider}

TMT PO design used 3 Spiders and 3 pairs of Tension Wires (Fig 5) to support the M2 system. Design change to adopt 6 Spiders and removing Tension Wires (Fig 6) was chosen given the following merits:

- Removal of Tension Wires avoids the variation in deformation caused by the large variation in the axial tension force during change in the EL angle.

- Relative displacement between M1 and M2 is reduced to be $1.99 \mathrm{~mm}$ in the observing EL range. 


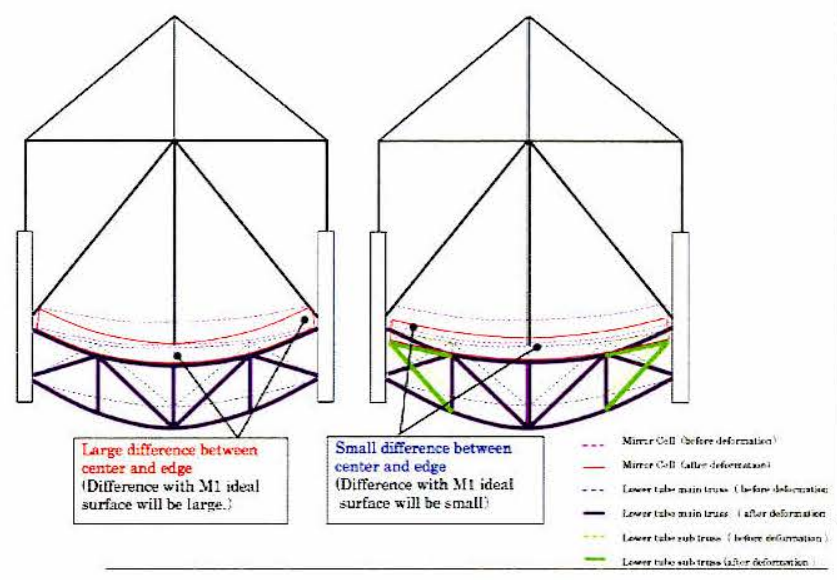

Figure 3. Double Lower Tube Structure (Conceptual Diagram of Deformation)

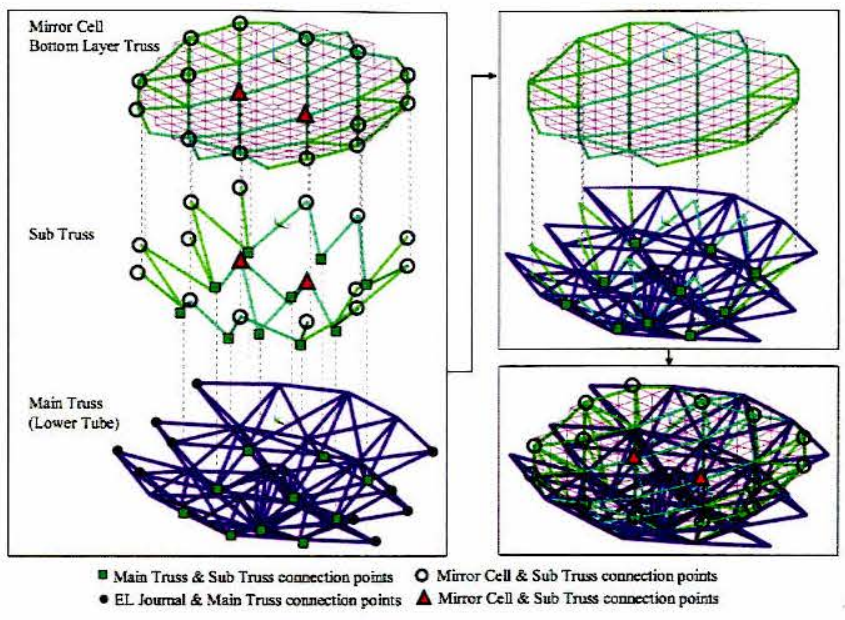

Figure 4. Double Lower Tube Structure (Configuration)

- Reduction of deformation of the Top Ring helps to reduce the deformation of the Lower Tube connected to Top Ring via Upper Tube resulting in relaxation of the required stroke for M1 actuators.

- Design change can be accomplished without increasing the optical vignetting.

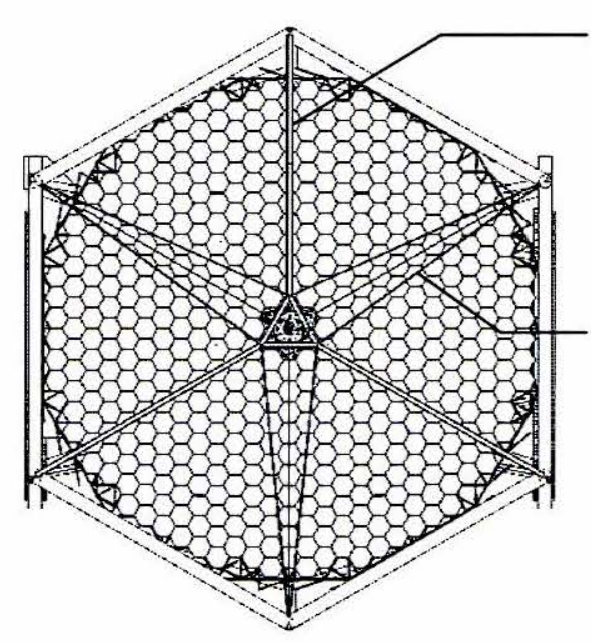

Figure 5. Baseline Design 3-Legged Spider (Width: 300 mm, Height: $600 \mathrm{~mm}$ )

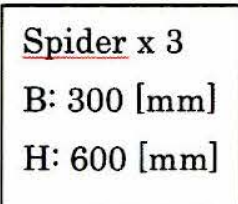

Tension wire $\mathrm{x} 6$

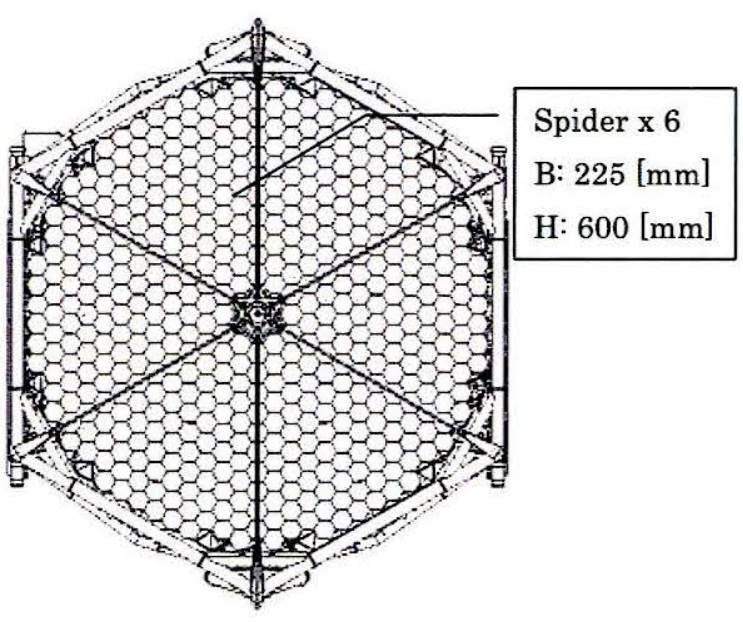

Figure 6. New MELCO Design 6-Legged Spider (Width: $225 \mathrm{~mm}$, Height: $600 \mathrm{~mm}$ )

\subsection{Risk Mitigation System against An Earthquake at a level of a 1,000 Year Return Period}

DRD defines the safety requirement for maximum acceleration allowable for M1, M2, M3, LGSF and Science Instruments as well as for STR uplift from the AZ and EL HSBs. Safety requirements apply for any orientation of telescope, regardless of the operational conditions of HSB, and Stow Lock Pins. Original approach to meet 
these requirements with assumed damping factor of $1 \%$ was challenging and serious efforts of iterating design changes and verifying the dynamic performances resulted in the addition of the following devices:

- XY Sliders underneath the STR AZ Structure (Fig 7) and implementation of an Early Earthquake Warning System which is currently in the planning stages and collaborated with the Hawai'i Volcano Observatory in order to avoid stress concentration when the HSB is not in operation

- AZ Rotation Brake to stop AZ rotational motion of the STR without constraining horizontal motion in the XY plane

- Damper System with optimum release of dampers to protect optics and instruments (Fig 7).

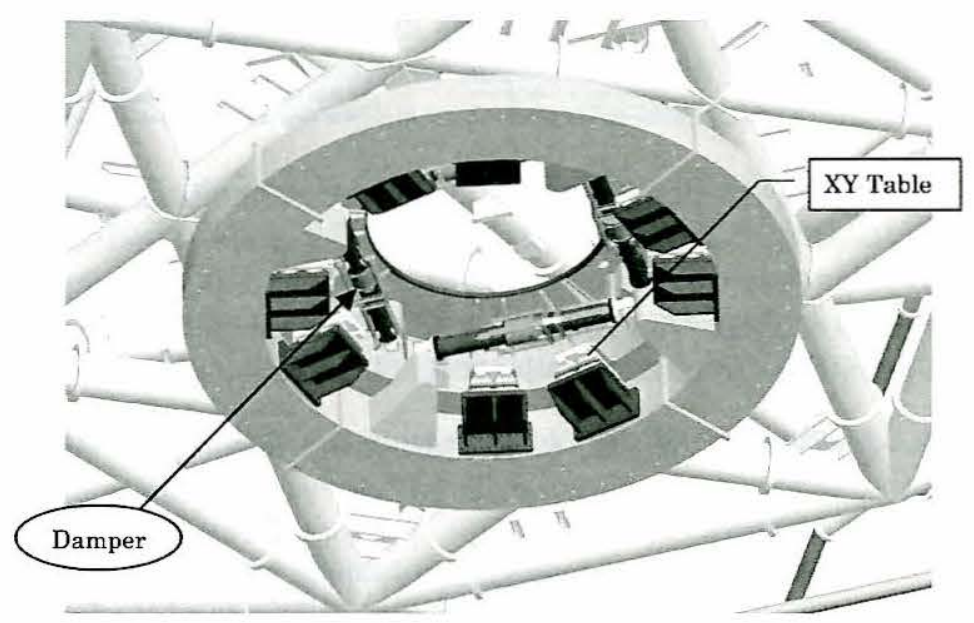

Figure 7. AZ Horizontal Seismic Isolation Dampers and XY Table (Rolling support and Uplift restraint)

In order to avoid cost increase, TMT PO decided to adopt a more practical damping factor of $2 \%$ instead of $1 \%$ for design safety and requested a simpler design option for seismic risk mitigation. The current design proposal for seismic risk mitigation assumes the following:

- HSB is operated all the time including daytime but with minimum necessary oil flow during the daytime to save on power consumption.

- Operation with STR not floating on an oil film is removed as a condition of operation so that additional XY Slides and Dampers, with exception for the dampers for the Pintle Bearing, can be removed, resulting in cost savings for those devices.

Seismic analyses were conducted for seven 1,000-year-return-period earthquakes at four EL angles of the telescope. Maximum acceleration value for each of the seven seismic waveforms were calculated to compare the average of those seven values with the specification. We confirmed that all optics, Science Instruments, and LGSF meet the specification (Fig 8).

\subsection{Telescope Pier Design assuming 1,000 MPa of Ground Soil Stiffness}

Design optimization of the Pier and AZ rail is made to satisfy the pointing performance requirement (cf. residual pointing errors caused by STR shall be less than 0.6 arcsec RMS) which is defined in DRD by evaluating the deformation of the pier taking the stiffness of the pier and ground into account under the concentrated load from HSB along the AZ thrust direction. The $1,945 \mathrm{~mm}$ width and $400 \mathrm{~mm}$ thick $\mathrm{AZ}$ rail is recommended to meet the requirements. The variation of the tracking error is is $0.176 \operatorname{arcsec}$ for an $\mathrm{AZ}$ complete rotation 


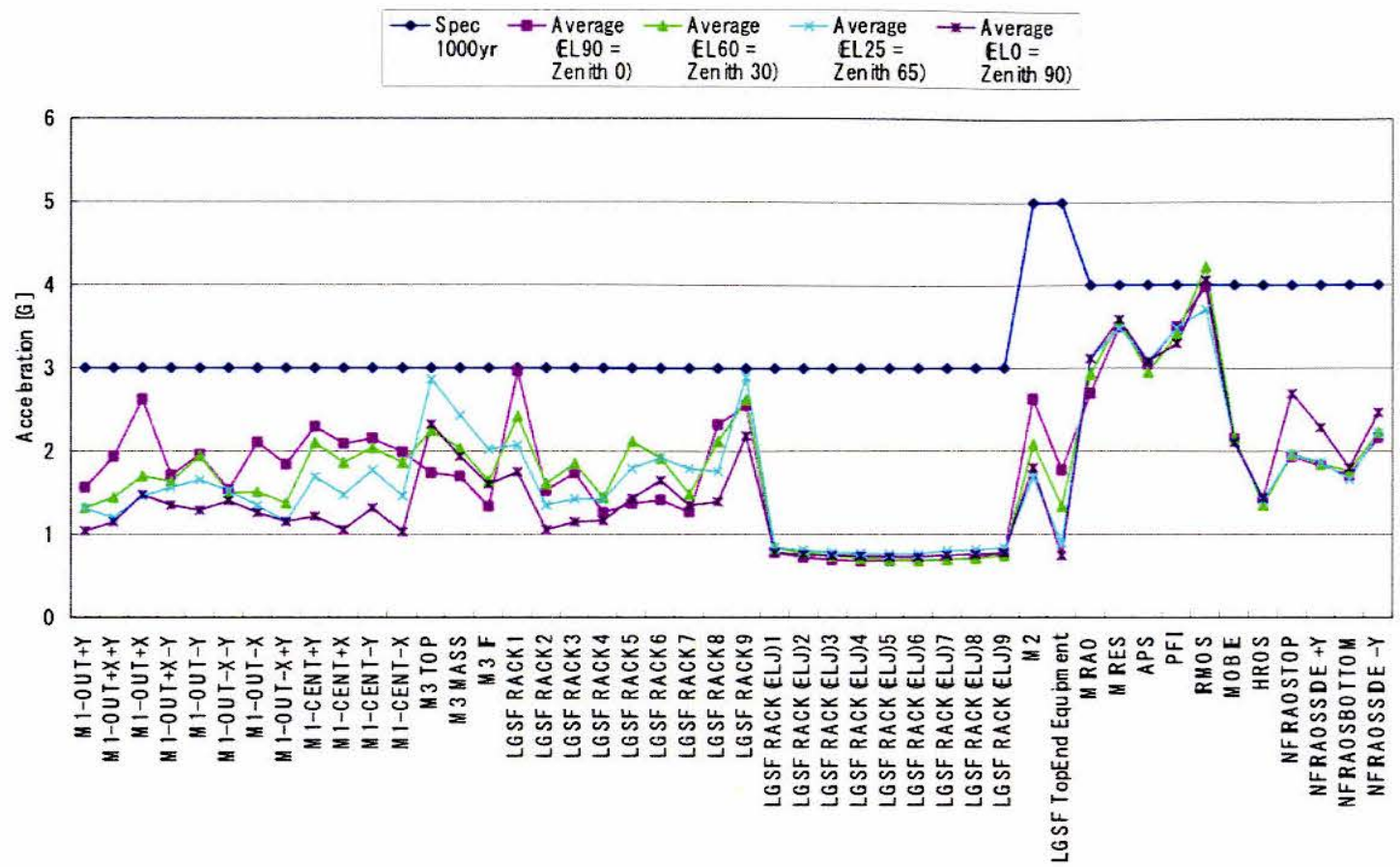

Figure 8. Maximum acceleration [G] of each component at various EL angles, averaged for seven seismic waveforms

which is significant with respect to the specification as it is 0.074 arcsec smaller than the baseline model $(1,200$ $\mathrm{mm}$ width). The size is also a convenient for the purpose of manufacturing, bolting and assembly. Ultra high strength concrete is also recommended as the material for the pier as the stress on the pier high regardless of its thickness. Soil stiffness has a very large affect on the rail displacement and the pier strength. The impact of the soil stiffness must be considered especially because the rail deformation value directly impacts the tracking error.

\subsection{Primary Mirror Segment Handling System (SHS)}

SHS is required to be able to make removing and installing operations of about 10 Mirror Segment Assemblies per day safely and with ease over the Primary Mirror area where access of personnel is extremely difficult. This requires semi-automatic sequence operation and a robotic Segment Lifting Fixture (SLF) is designed based on the Compliance Control System (Fig 9 and 10) with a mechanism to enable precise control within the 6 degrees of freedom of position control.

As the relative positioning of the Segment Lifting Fixture and Mirror Segment Assembly should be achieved without relying on a vision of a person, image analysis system (Machine Vision) is used to control the operation.

\section{6 $\mathrm{CO}_{2}$ Cleaning Mechanism}

In the baseline design, three CFRP arms are rotated along M1 with their tips hung and pulled by wires within the horizontal plane (X,Y plane). Each of the 3 arms rotate by 120 degree like a windshield wiper to cover the whole M1 area. The arm storage positions shall not vignette the optical beam but there are unresolved problems, such as the SHS bridge colliding with the arm storage during rotation and the arm rotating wires vignetting the beam. In order to resolve the issues, the use of wires was abandoned in favor of employing four truss arms which are bent to avoid vignetting when stored. In addition, their rotating axes are moved to the four corners of M1 in order to avoid interference with the SHS bridge. The issue with interference with other structures is resolved although the number of arms is increased from three to four that the similar mechanical system has been used at 


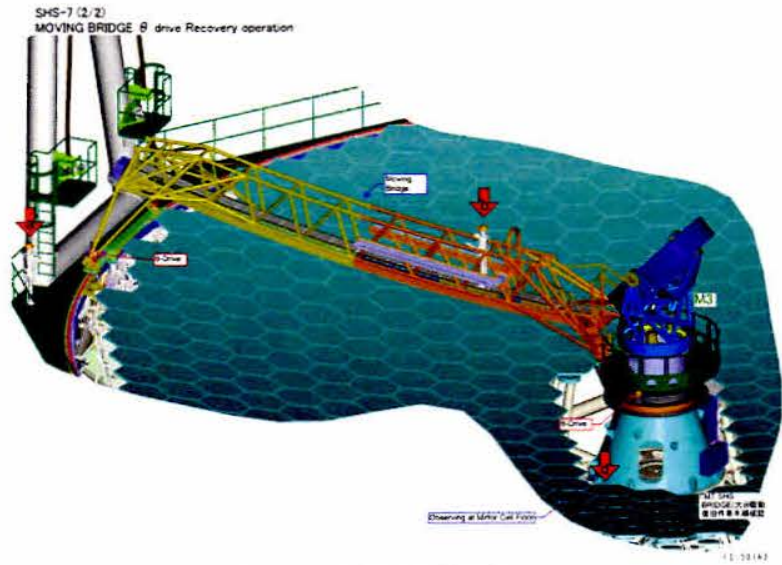

Figure 9. SHS Bridge

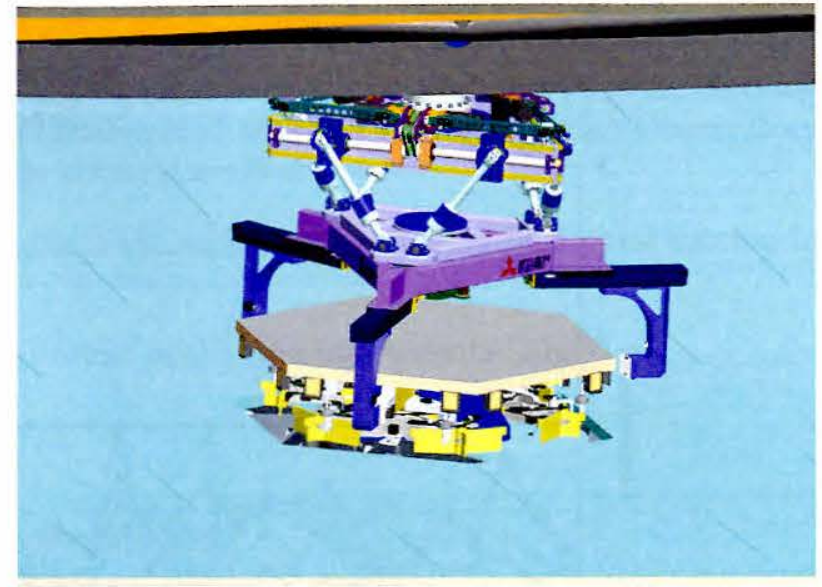

Figure 10. Segment Lifting Fixture (SLF) with Lifting Talons to hook M1 segment mirror

Subaru Telescope (Fig 11). The four arms are adopted as higher priority is placed on the prevention of collision with other structures. The arms are optimally arranged as a whole within this restriction (Fig 12).

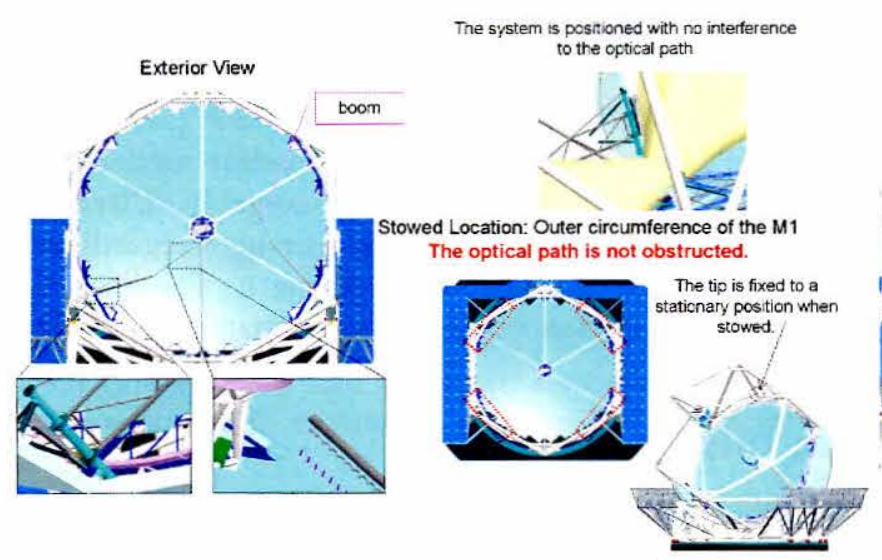

Figure 11. Four $\mathrm{CO}_{2}$ Moving Arm

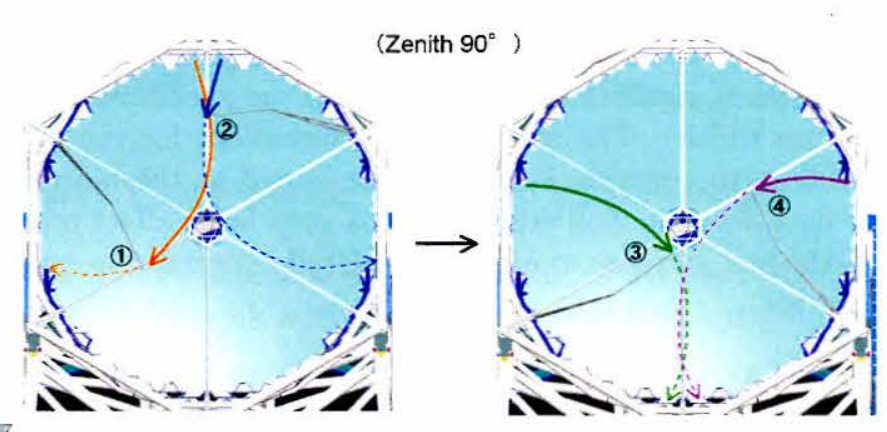

Figure 12. $\mathrm{CO}_{2}$ Arm moving pattern for M1 cleaning

\subsection{AZ Stopping System without Hardware Stops}

The following system is proposed to remove AZ hardware stoppers while securing a method for stopping AZ rotation safely for seismic events. In order to secure seismic isolation, AZ brakes should not be engaged even when power failure takes place because the brakes translate the seismic vibration to the telescope structure. However, adoption of a brake system with such a clamp releasing function is not compatible with the requirement for safe stopping of $\mathrm{AZ}$ overrun. For this reason, redundant limit switches to detect overrun and dynamic brakes together with hydraulic brakes are employed to realize a system for stopping the AZ movement safely by reconciling the two requirements for safety. With this system, overrun is prevented and the cable wrapping system is protected.

\subsection{Cable Wrapping System}

TMT' s design locates cable trays on additional horizontal pipe structures between AZ center and Nasmyth platforms. New design does not employ additional pipe structure but uses main structure pipes as fixture points for the cable trays. This design enables lighter weight and better accessibility to the cables for the purpose of 


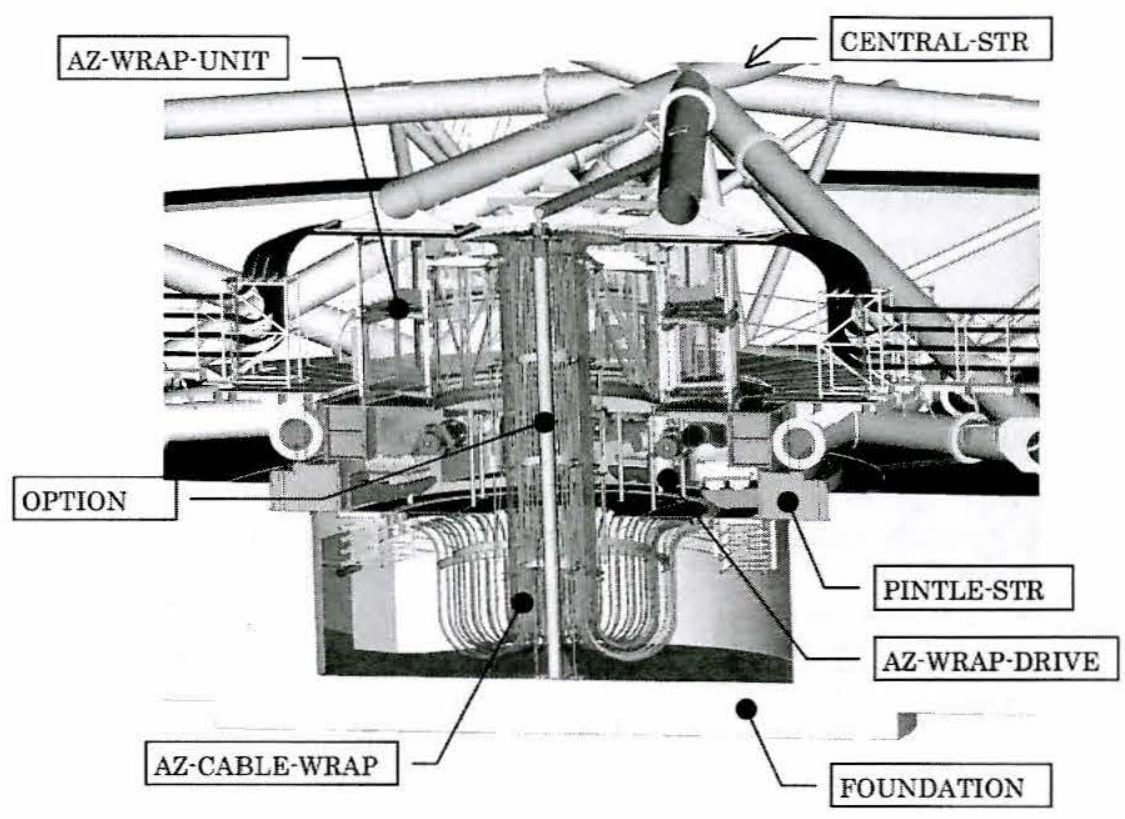

Figure 13. AZ Active Cable Wrapping System

maintenance. An active cable wrapping system is employed in order to reduce effects of reaction forces due to cable wrappings on the pointing errors. Active cable wrapping systems rotate by themselves synchronously to the AZ structure and hardly translate the reaction forces of cables and pipes to the structure. With a minimized radius of curvature of the cable wrapping system, their rotatable range are extended. In addition, cableveyors supported by ball bearings are employed in order to further reduce the reaction forces (Fig 13).

\subsection{M3 Tower}

A cone-shaped cylindrical structure was employed for the M3 tower instead of the truss structure in the baseline design in order to increase the rigidity for earthquakes and self-gravity deformation. Because the M3 unit is supported at six points by the M1 truss, an additional structure for conversion from hexagon to circular plate may reduce the rigidity. However, a cone shaped structure made of thin plates is employed to simultaneously attain high rigidity and lighter weight. This structure allows for efficient circle to circle connection to the M3 tower unit (Fig 14).

Accessibility to the SHS bridge is also improved with a path to the M3 unit through the central part of the cone-shaped structure. This structure can provide further merits by allowing deformation to be reduced by the use of shims for angle adjustment at the root of the cone-shaped structure.

\subsection{Mechanical interfaces for Science Instruments, NFIRAOS, and LGSF}

Many hard points are provided in the grid configuration at the Nasmyth platform to enable deployment of future science instruments. Such a systematic design provides stable configuration for science instruments and even larger flexibility in planning the deployment of future science instruments. Detail mechanical interfaces for the first generation science instruments have not been fixed yet. Detailed studies will be made upon ICDs becoming available. A study has been performed for the basic principle of placing numerous hard points in grids on the Nasmyth platform. This design provides freedom to fit future unknown instruments and minimizes deformation due to load changes. The LGSF layout is modified to avoid the interference between the additional structural pipes needed for the "Double Lower Tube" structure, LGSF optical amplifier installed inside EL Journal, and the control rack. Although the change in the layout will make carrying out the control racks for maintenance more difficult, the work can still be completed by utilizing jigs for that purpose. A wider floor and passage space is secured and accessibility is improved. The design for the doors is also modified to improve serviceability. 


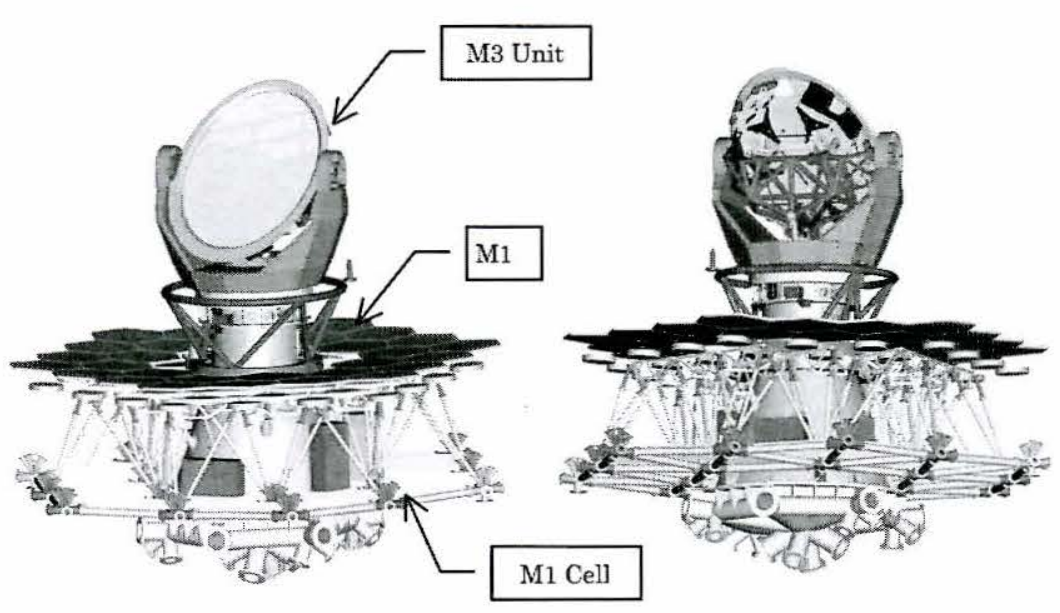

Figure 14. M3 Tower Structure

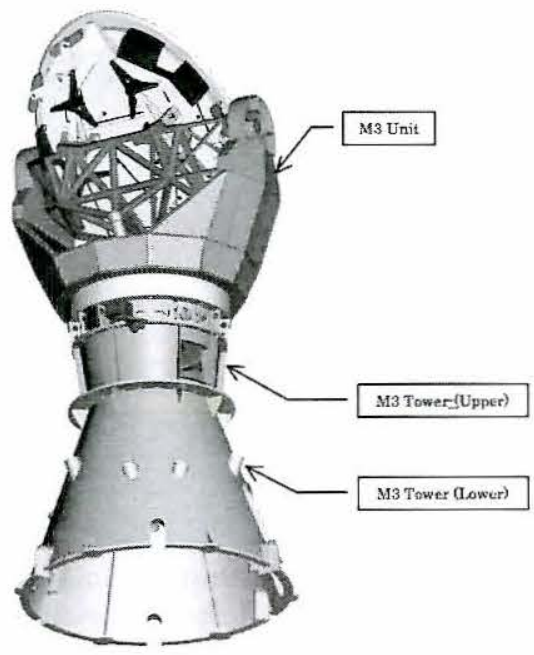

\section{PRELIMINARY DESIGN REVIEW AND CURRENT STATUS}

The Preliminary Design Reviews (PDR) for STR including its Control Systems were held on November 12-14, 2013 and April 15-16, 2014 in Japan. The major objectives of the PDR were to determine whether the following have all been adequately addressed:

- Recommendations of the CoDR Panel Report have been addressed;

- All significant design choices are made;

- The design and interface requirements are met;

- The design is structurally efficient and cost effective;

- Enabling technologies have been developed;

- Major risks have been identified and are being managed;

- Safety hazards have been analyzed and are being mitigated;

- Plans and schedules have been developed for fabrication, long-lead time procurement, AIVs;

- Bottom-up fabrication and construction cost estimates have been developed;

- Schedule and cost of the next phase are understood;

- The management plan is appropriate to control the work.

The international review panels recognized that MELCO and NAOJ have completed extensive design work in advancing the telescope structure design for Conceptual Design level to Preliminary Design level and the current design exceeds Preliminary Design level (e.g. on-site construction design). The preliminary design works were successfully approved by the panel and the works are now moving to the final design phase from April 2014 for two years.

\section{ACKNOWLEDGMENTS}

We gratefully acknowledge the support of the TMT collaborating institutions. They are the Association of Canadian Universities for Research in Astronomy (ACURA), the California Institute of Technology, the University of California, the National Astronomical Observatory of Japan, the National Astronomical Observatories of China and their consortium partners, and the Department of Science and Technology of India and their supported institutes. This work was supported as well by the Gordon and Betty Moore Foundation, the Canada Foundation for Innovation, the Ontario Ministry of Research and Innovation, the National Research Council of Canada, the Natural Sciences and Engineering Research Council of Canada, the British Columbia Knowledge Development Fund, the Association of Universities for Research in Astronomy (AURA), the U.S. National Science Foundation and the National Institutes of Natural Sciences of Japan. 